\title{
Sensibilidad del Eco Doppler en el Trauma Vascular de Miembros Inferiores. Experiencia en el Hospital 'Dr Domingo Luciani'
}

\section{Carlos Nuñez, Edward Cabrera, Patricia Torres, Tamarys Cova, Omar Castro}

\section{RESUMEN}

Objetivo general: Determinar la sensibilidad del Eco Doppler en las lesiones vasculares por trauma de miembros inferiores en pacientes ingresados con esta impresión diagnóstica en el Hospital Dr Domingo Luciani durante el período Abril 2008-Diciembre 2011.

Objetivos específicos: Determinar Grupo etario y sexo del paciente con lesión vascular. Definir el mecanismo de trauma de los pacientes sometidos a Eco Doppler por lesión vascular. Mencionar los hallazgos ecográficos de los pacientes ingresados con lesión vascular. Conocer la lesión vascular más frecuente. Precisar los hallazgos quirúrgicos de los pacientes sometidos a Eco doppler por lesión vascular. Correlacionar los hallazgos quirúrgicos con los ecográficos.

Método de investigación: Estudio retrospectivo, descriptivo. Basado en la base de datos de la Unidad de Politraumatizados y en los Servicios de Cirugía General del hospital 'Dr Domingo Luciani'. Lapso 2008-2011.

Criterios de inclusión: Paciente mayor de 18 años con impresión diagnóstica de lesión vascular de miembros inferiores por trauma. Eco Doppler realizado por las mismas residentes del Servicio de Radiodiagnóstico del Hospital.

Criterios de exclusión: Pacientes con lesión vascular de miembros superiores. Pacientes hemodinámicamenteinestables. Eco Doppler realizado por residentes del Servicio de Radiodiagnósticos no incluidos en la investigación.

Palabras claves: Trauma, EcoDoppler.

\section{ABSTRACT}

Introduction and objectives: To determine the accuracy of Doppler vascular test in lower limb trauma patients admitted to Dr Domingo Luciani Hospital during the period April 2008 to December 2011.

Materials and methods: A retrospective, descriptive. Based on the database of the polytrauma unit and General Surgery Services Hospital 'Dr Domingo Luciani'. Inclusion criteria: Patients over 18 years with vascular injury diagnostic impression of lower limb trauma.

Results: A total of 27 patients received emergency lower limb vascular injury, being most common in men $85 \%$ (23 cases), with a mean age of 23.3 years, trauma mechanism by gunshot wound of $70.3 \%$ (19 cases ), stab $22.22 \%$ (6 cases) and musculoskeletal trauma closed $7.5 \%$ (2 cases), the most common ultrasound finding was the presence of single phase flow $81.4 \%$ (22 cases), vascular injury seen most commonly intraoperatively was laceration $59.2 \%$ (16 cases), the vessel was found to be more injured superficial femoral artery $48.14 \%$ (13 cases), $14.81 \%$ (4 cases) reported no evidence of vascular injury surgically as finding. One case $(3.7 \%)$ was negative on Doppler but was found positive at operation.

Conclusion: Doppler ultrasonography has greater sensitivity than specificity for the diagnosis of vascular lesions in the lower limbs.

Keywords: Trauma vascular, Eco Doppler.

How to cite this article: Nuñez C, Cabrera E, Torres $P$, Cova T, Castro O. Sensibilidad del Eco Doppler en el Trauma Vascular de Miembros Inferiores. Experiencia en el Hospital 'Dr Domingo Luciani'. Panam J Trauma Critical Care Emerg Surg 2012;1(3):159-162.

Source of support: Nil

Conflict of interest: None declared

\section{INTRODUCCIÓN}

Los traumatismos vasculares en la vida civil han aumentado de forma importante; Esto se debe al incremento de los accidentes de circulación, laborales y domésticos, al aumento de la violencia en nuestra sociedad y al creciente número de complicaciones vasculares iatrogénicas. El trauma vascular tiene una alta incidencia dentro de la población masculina juvenil, con una franja etaria entre los 20 y 40 años de edad. La violencia y la práctica quirúrgica actual han traído consigo un incremento notable de los problemas para la cirugía de trauma, por lo que se ha mantenido una búsqueda constante del conocimiento, de la fisiopatología del trauma, con la consecuente mejoría del manejo adecuado del paciente politraumatizado. $^{1}$

El trauma vascular sigue siendo un reto para los cirujanos; Desde un punto de vista quirúrgico los traumatismos vasculares se dividen en abiertos y cerrados. En el traumatismo abierto existe siempre una contaminación bacteriana de la herida que condiciona el tratamiento y la evolución. Morfológicamente, podemos clasificar a las heridas vasculares en: punciones, laceraciones, secciones, contusiones, avulsiones, compresiones, fístulas

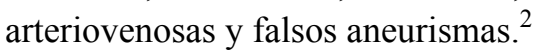

En muchos casos el diagnóstico es obvio, sobre todo en presencia de heridas profundas situadas sobre el trayecto de los vasos o cuando se observa una franca hemorragia a través de ellas. En otras ocasiones el diagnóstico puede no ser tan evidente e incluso estar enmascarado por las lesiones asociadas. En todo caso es necesario un alto grado

Paper presented at the resident competition, 25th Annual Congress of Panamerican Trauma Society, Medellin, Colombia, November 2012. 
de sospecha para hacer el diagnóstico correcto o hacer las exploraciones adecuadas que lleven al mismo. En toda herida próxima al recorrido de los vasos se debe excluir su lesión, sobre todo si se acompaña de hematomas expansivos o pulsátiles y de hemorragia copiosa. ${ }^{6}$

El diagnóstico de lesiones importantes, se relaciona con la ausencia de pulsos en el examen físico, por lo tanto la presencia de señal al doppleren una extremidad sin pulsos, sólo da una sensación de falsa seguridad y no confirma la ausencia de lesión. Frente a un pulso palpable, pero disminuido en el dopplerpuede orientar al clínico, pero tampoco certifica la ausencia de lesión, este examen es muy operador dependiente. La utilización de aparatos de ultrasonidos (Doppler o Eco- Doppler) para explorar el flujo vascular es de gran ayuda, sobre todo en pacientes hipotensos con pulsos difíciles de palpar. ${ }^{4}$

Cuando existe sospecha de un traumatismo arterial la exploración de elección es la arteriografía. Su realización, una vez controladas las alteraciones que amenazan la vida del traumatizado, no complica la evolución y retrasa poco o incluso adelanta el tratamiento adecuado. En pacientes en estado grave que requieren una operación quirúrgica inmediata, la arteriografía puede ser realizada en quirófano pues no son necesarias instalaciones especiales. En las extremidades las radiografías son importantes, ya que algunos tipos de fractura en el tercio distal del fémur o proximal de la tibia se asocian con frecuencia con lesiones vasculares. Lo mismo ocurre en las fracturas próximas a la articulación del codo. ${ }^{1}$

Se considera que la alta frecuencia de lesiones en los vasos femorales está en relación con la extensión de estos y lo relativamente poco protegidos y superficiales que transcurren al nivel del muslo. Se conoce también que aunque el trauma vascular periférico por arma de fuego es el más frecuentes, la localización en las extremidades inferiores se dice que es porque aun cuando predomina el deseo de defenderse nadie pretende provocar lesiones mortales, algo común en lo que se ha denominado la guerra urbana en ciudades sobre todo de Colombia y Estados Unidos de América. Los traumas vasculares representan una preocupación para el cirujano que se los trata, la necesidad de un tratamiento oportuno, completo y a la vez rápido exige destreza y conocimiento del problema. ${ }^{4}$

Tabla 1: Frecuencia del trauma vascular en miembros inferiores según el género

\section{Género}

Masculino

Femenino Casos

23

4 Porcentaje 85 15

Fuente: Base de datos unidad politraumatizados, Hospital Domingo Luciani 2008-2011

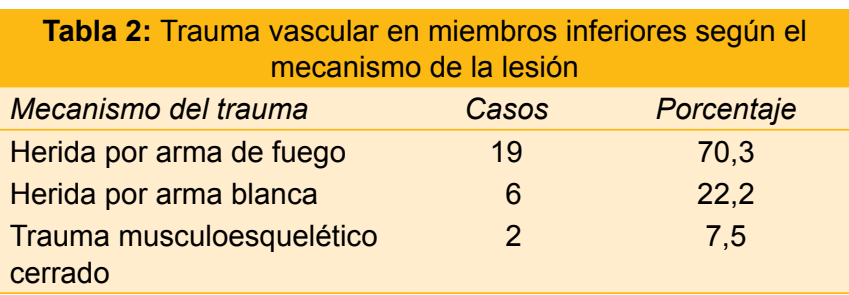

Fuente: Base de datos unidad politraumatizados, Hospital Domingo Luciani 2008-2011

Tabla 3: Hallazgos ecográficos en el trauma vascular de miembros inferiores

\begin{tabular}{lcc} 
Hallazgo ecográfico & Casos & Porcentaje \\
\hline Flujo monofásico & 22 & 81,4 \\
Ausencia de flujo & 4 & 14,8 \\
Flujo normal & 1 & 3,7
\end{tabular}

Fuente: Base de datos unidad politraumatizados, Hospital Domingo Luciani 2008-2011

\begin{tabular}{|c|c|c|}
\hline Lesión vascular & Casos & Porcentaje \\
\hline Contusión & 1 & 3,7 \\
\hline Espasmo & 3 & 11,1 \\
\hline Laceración & 1 & 3,7 \\
\hline Transección incompleta & 16 & 59,3 \\
\hline Transección completa & 6 & 22,2 \\
\hline
\end{tabular}

Fuente: Base de datos unidad politraumatizados, hospital domingo Luciani 2008-2011

\begin{tabular}{|c|c|c|}
\hline Vaso & Casos & Porcentaje \\
\hline Arteria femoral superficial & 13 & 48,1 \\
\hline Arteria femoral profunda & 6 & 22,2 \\
\hline Arteria poplítea & 5 & 18,6 \\
\hline Tronco tibioperoné & 1 & 3,7 \\
\hline Arteria tibial anterior & 1 & 3,7 \\
\hline Arteria tibial posterior & 1 & 3,7 \\
\hline
\end{tabular}

Fuente: Base de datos unidad politraumatizados, hospital Domingo Luciani 2008-2011

\begin{tabular}{|c|c|c|c|}
\hline \multirow[t]{2}{*}{$\begin{array}{l}\text { Hallazgos } \\
\text { ecosonográfico }\end{array}$} & \multirow[t]{2}{*}{ Casos } & \multicolumn{2}{|c|}{$\begin{array}{c}\text { Exploración vascular } \\
\text { casos }\end{array}$} \\
\hline & & $\begin{array}{l}\text { Con lesión } \\
\text { vascular }\end{array}$ & $\begin{array}{l}\text { Sin lesión } \\
\text { vascular }\end{array}$ \\
\hline Con lesión vascular & 26 & 23 & 3 \\
\hline Sin lesión vascular & 1 & 1 & 0 \\
\hline Total & 27 & 24 & 3 \\
\hline
\end{tabular}

Fuente: Base de datos unidad politraumatizados, Hospital Domingo Luciani 2008-2011

\section{RESULTADOS}

Un total de 27 pacientes fueron intervenidos de emergencia por lesión vascular en miembros inferiores, siendo lo más 
frecuente en hombres $85 \%$ ( 23 casos), con edad promedio de 23,3 años, mecanismo del trauma por herida de arma de fuego $70,3 \%$ (19 casos ), arma blanca $22,22 \%$ (6 casos) y trauma musculoesquelético cerrado $7,5 \%$ ( 2 casos), el hallazgo ecográfico más común fue la presencia de flujo monofásico $81,4 \%$ (22 casos ), la lesión vascular evidenciada intraoperatoria más frecuente fue transección incompleta $59,3 \%$ (16 casos), el vaso más lesionado resultó ser la arteria femoral superficial 48,14\% (13 casos), el 11,11\% ( 3 casos) reportó lesión vascular (posible vasoespasmo) sin evidenciarse quirúrgicamente tal hallazgo y $3,7 \%$ ( 1 caso) se concluyó por Doppler no tener lesión vascular, sin embargo intraoperatorio se pudo observar la misma (contusión).

\section{DISCUSIÓN}

El trauma vascular periférico, sigue siendo un importante problema de salud publica, que día a día va en ascenso, el valor del diagnóstico temprano para una terapéutica oportuna también es de vital importancia, ya que la detención precoz de una lesión vascular en un miembro permite su pronta revascularización en un tiempo menor a las 6 horas del trauma. $^{3}$

La frecuencia del trauma vascular periférico, en el presente estudio, reporto $85 \%$ de afectación al genero masculino y un $15 \%$ para el femenino; (ver Tabla 1). Dicho resultado se asemeja a los encontrados en ciertas publicaciones, como por ejemplo el estudio de (Soteras 2010); que analizaron 470 pacientes con lesión vascular en extremidades superiores e inferiores, $86,6 \%$ corresponde al genero masculino y $13,4 \%$ al femenino. Cuando analizamos el mecanismo de la lesión se pudo determinar que los traumatismo en extremidades que condicionan una afectación de tipo vascular, el arma de fuego se encuentra en un 70,3\% en frecuencia (ver Tabla 2), un 22,2\% corresponde al arma blanca (ver Tabla 2); y un bajo porcentaje se le asigna al trauma musculoesquelético cerrado con tan solo 7,5\% (ver Tabla 2); en el estudio de (soteras 2010); cuyo mecanismo lesional en un $69,5 \%$ fue trauma penetrante y $30,5 \%$ corresponde a trauma cerrado, en el grupo de trauma penetrante el arma de fuego predominó en 243 casos de los 326 existentes, es así, como el trauma vascular llega hacer un problema de salud pública.

Cabe destacar, que dentro de los métodos diagnósticos del trauma vascular, el factor imperante es la clínica, donde se evalúan los signos blandos y duros de lesión vascular, como método alternativo confirmatorio, en pacientes con estabilidad hemodinámica, se encuentra el eco-Doppler, en el presente estudio; se logro evidenciar que de los 27 pacientes evaluados, en un $81,4 \%$ la conclusión ecográfica fue flujo monofásico distal a la lesión (ver Tabla 3), en un
$14,8 \%$ reportó ausencia de flujo (ver Tabla 3), y en un 3,7\% reportó flujo normal, que es el equivalente a 1 caso, cabe destacar, que el eco-doppler no es más que una combinación del estudio hemodinámico de la velocidad del flujo y el estudio morfológico del vaso afectado. ${ }^{5}$

Cuando se produce la lesión vascular, podemos evidenciar intraoperatorio los siguientes tipos de lesiones; contusión, espasmo, laceración, transección incompleta y transección completa, en la siguiente revisión se logró encontrar, que un 59,3\% presentótransección incompleta del vaso afectado (ver Tabla 4), un $22,2 \%$ presentótransección completa (ver Tabla 4), en un $11,1 \%$ se evidencio vasoespasmo, un 3,7\% se le asigna a la contusión y un mismo valor para la laceración, muchos estudios reportan manejo conservador de lesiones tipo vasoespasmos. Estenosis pequeñas, previamente determinadas por estudios angiográficos, posterior a la realización del eco-Doppler. ${ }^{7}$

Con respecto, al vaso lesionado en el trauma vascular periférico, en dicho estudio se determinó que la arteria femoral superficial fué la más afectada, para un valor porcentual de $48,1 \%$, (ver Tabla 5), la arteria femoral profunda un $22,2 \%$ (ver Tabla 5), arteria poplítea $18,6 \%$ (ver Tabla 5), tronco tibioperoneo 3,7\% (ver Tabla 5), arteria tibial anterior 3,7\% (ver Tabla 5), arteria tibial posterior 3,7\%; (ver Tabla 5); la arteria femoral superficial por su disposición anatómica, tiene mas tendencia a lesionarse, en la mayoría de los estudios publicados existe un alto porcentaje de trauma asignado a dicho vaso, en un estudio con 236 casos de lesión vascular de miembro inferior, 130 casos correspondían a lesión de la arteria femoral superficial para un valor de $53,2 \%{ }^{6}$

Si correlacionamos los hallazgos ecosonográficos con los quirúrgicos, al realizar la exploración vascular, se pudo evidenciar en dicho estudio que; 26 pacientes que fueron llevados a mesa quirúrgica tenían un eco-Doppler que concluíalesión vascular, siendo acertado este diagnóstico en 23 pacientes que fueron sometidos a cirugía, 3 de ellos no reportólesión vascular intraoperatoria, es así, que 1 solo paciente reportó hallazgos ecosonográficos negativos para lesión vascular, pero imperando la clínica se lleva amesa quirúrgica y se evidencia lesión, por tanto, existe un $88,46 \%$ de correspondencia entre los pacientes que tenían un eco-doppler positivo que seevidencio en el acto quirúrgico; valor que se asemeja a la sensibilidad del eco-Doppler un $87 \%$ (Table 6$)^{2}$

\section{CONCLUSIONES}

- En el trauma vascular periférico el sexo masculino fue el predominante. 
- El mecanismo de trauma más común fue el arma de fuego.

- El hallazgo ecosonográfico más común fue el flujo monofásico.

- La lesión intraoperatoria mas frecuente fue la transección incompleta y la arteria lesionada más común fue la femoral superficial.

- El eco demostró ser una herramienta importante sin embargo sigue siendo operador dependiente.

\section{REFERENCIAS}

1. Anderson, Robert y otros: Reduced dependency on arteriography for penetrating extremity trauma. J Trauma Sept 2008, N ${ }^{\circ} 34$ pág. 1096.

2. Pereira LM. Epidemiologia de trauma vascular. Rev Esp 2009 Vol. 4 pág. 15

3. Fernando RM. Trauma vascular periférico. Manejo del paciente politraumatizado 2008.

4. Cristian SD. Trauma vascular, vision cirujano cardiovascular. Rev Med Clin Condes 2011;22(5):686-96.

5. SherifAA. Vascular injuries. Int Surg 2008 Apr-Jun;77(2):114-17.

6. Soteras J, et al. Trauma vascular. Nuestra experiencia en 10 años. Rev Argentina de Cirugía Cardiovascular 2010 Marzo-abril.
7. Lynch K, et al. Can Doppler pressure measurement replace, exclusion arteriography in the diagnosis of occult extremity arterial trauma. Surg 2009;214:717.

\section{ACERCA DE LOS AUTORES}

\section{Carlos Nuñez (Correspondiente Autor)}

Cirujano General, Hospital 'Dr Domingo Luciani', e-mail: edalex81@ gmail.com

\section{Edward Cabrera}

Cirujano General, Hospital 'Dr Domingo Luciani'

\section{Patricia Torres}

Residente, Hospital 'Dr Domingo Luciani'

\section{Tamarys Cova}

Residente, Hospital 'Dr Domingo Luciani'

\section{Omar Castro}

Residente, Hospital 'Dr Domingo Luciani' 